\title{
Joint moments of proper delay times
}

\author{
Angel M. Martínez-Argüello and Moisés Martínez-Mares \\ Departamento de Física, Universidad Autónoma Metropolitana-Iztapalapa, \\ Apartado Postal 55-534, 09340 México Distrito Federal, Mexico \\ Julio C. García \\ Departamento de Matemáticas, Universidad Autónoma Metropolitana-Iztapalapa, \\ Apartado Postal 55-534, 09340 México Distrito Federal, Mexico
}

(Dated: July 22, 2018)

\begin{abstract}
We calculate negative moments of the $N$-dimensional Laguerre distribution for the orthogonal, unitary, and symplectic symmetries. These moments correspond to those of the proper delay times, which are needed to determine the statistical fluctuations of several transport properties through classically chaotic cavities, like quantum dots and microwave cavities with ideal coupling.
\end{abstract}

\section{INTRODUCTION}

The generalized Laguerre ensemble appears in the context of chaotic scattering 1,2 as being the joint distribution of the reciprocals of the eigenvalues of the Wigner-Smith time delay matrix, known as the proper delay times.

The Wigner-Smith time delay matrix was introduced by Smith ${ }^{3}$ as a multi-channel generalization of the concept of delay time suffered by a wave packet, due to the interaction with a scattering potential, introduced by Wigner in the one channel situation. ${ }^{4}$ It is an $N \times N$ Hermitian matrix, where $N$ is the number of open modes (or channels), whose eigenvalues, the proper delay times, represent individual delay times on the channels $\frac{1.2}{n}$ This time delay matrix is defined in terms of the energy derivative of the scattering matrix $S$, which is a fundamental entity in the description of scattering processes, and many transport properties in open systems, by relating the outgoing plane wave amplitudes to the incoming ones into the $N$ channels. ${ }^{\frac{5}{2}}$ Therefore, the Wigner-Smith time delay matrix is very important in the quantification of the transport properties which depend on the derivative of the scattering matrix with respect to the energy or an external parameter. The activity in this field has been increased due to the recent theoretical investigations $\underline{\underline{6}-\underline{\underline{9}}}$ that emerged from a measurement of a delay time in experiments of interaction of light with matter, with attosecond precision $\frac{10}{2}$

In chaotic scattering, one of the most important questions is the effect of the chaotic classical dynamics of open ballistic cavities on the transport properties (see 11 14 and references therein). For example, the parametric derivative of the conductance through a quantum dot, which is the analogous to the level velocity in the characterization of a mesoscopic system, $15-18$ fluctuates with respect to some external parameters, that could be an applied magnetic

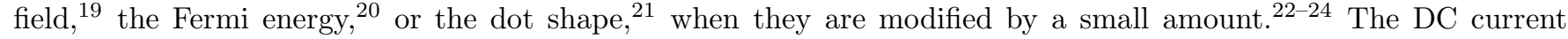
pumped through a quantum dot at zero bias is quantified by its fluctuations with respect to an applied magnetic field. ${ }^{25,26}$ The same situation occurs with the parametric derivative of the transmission coefficient, with respect to the frequency and cavity shape, in classical wave cavities $\stackrel{27,28}{2}$ The statistical analysis needed for the quantification of a transport property, or its fluctuation, is very well realized by the random-matrix theory, that reveals the universal aspects of the problem: $\stackrel{11-13}{\underline{n}}$ Since all of these quantities are defined in terms of the derivative of the scattering matrix this theory leads to quantify the transport properties, or their fluctuations, by the first moments of the proper delay times $\tau_{i}$ 's $(i=1, \ldots, N) \underline{24,27}-29$ In other cases, it is the distribution of the Wigner time delay, defined as the average of the proper delay times, what is of interest. For example, it is related to the dimensionless capacitance of a mesoscopic capacitor $\stackrel{30,31}{3}$ or to the thermopower $\stackrel{2}{2}$ in disordered systems it is used to characterize the classical diffusion in the metallic regime, $\stackrel{32}{2}$ the eigenfunction fluctuations (see Ref. 33 and references therein), and the metal-insulator transition $\underline{34,35}$

A lot of work concerning statistical studies of delay times and Wigner time delay has been developed in the last thirty years in several contexts of chaotic and disordered systems $\underline{2,31,36,37,39} \underline{43}$ For closed chaotic systems, those with non perfect coupling to the $N$ open channels, the mean and variance of partial or phase-shift times, being the energy derivative of the eigenphases of the scattering matrix, as well as of the Wigner time delay, are well known in absence of time reversal symmetry $(\beta=2), 36,37$ The existing distribution for partial delay times for arbitrary $N$ are given in Refs. 37, 38, and 41 for $\beta=2$, and in Ref. 41 in the presence of time reversal symmetry $(\beta=1)$ and spin-rotation symmetry $(\beta=4)$. Besides, the distribution of the Wigner time delay was first calculated in Refs. 30,37 for the $N=1$ case, while the one for $N=2$ and $\beta=2$ was calculated, generalized, and verified to arbitrary $\beta$ in the ideal coupling case, and related to that of arbitrary coupling in Ref. 41. The joint distribution of the reciprocals of the proper delay times, which is the Laguerre distribution, is also known for arbitraries $N$ and $\beta, 1,2,41$ However, it does not exist enough evaluations of the moments of this distribution for any symmetry and $N$. Expressions for the density 
of proper delay times and uncorrelated moments have been evaluated in Ref. 44 in the limit of very large $N$ and, for few and large number of channels and any symmetry, in Refs. 45. 46 more recently, both cases for perfect coupling.

Our purpose in the present paper is to evaluate several joint moments of proper delay times for any symmetry, and determine their dependence with the number of channels, when this number is arbitrary. Many of these moments have not been calculated before, some of which have also importance on transport properties through ballistic open systems. Therefore, we regard perfect coupling to the $N$ open channels.

In the next section we introduce the Laguerre distribution and establish the calculation of the moments we are interested in, in a general form, and summarize some known results for the partial times. In Sect. III we present some definitions and properties that will help us to manage the Laguerre distribution, and allow us to determine the moments for arbitrary $N$ and any symmetry. Explicit calculations are performed in Sect. IV. We conclude in Sect. V.

\section{DELAY TIMES AND GENERALIZED LAGUERRE DISTRIBUTION}

\section{A. Proper delay times}

A symmetrized form of the Wigner-Smith time delay matrix ${ }^{1}$ can be written in dimensionless units as

$$
Q=-\frac{\mathrm{i} \hbar}{\tau_{H}} S^{-1 / 2} \frac{\partial S}{\partial E} S^{-1 / 2}
$$

where $E$ is the energy and $\tau_{H}$ is the Heisenberg time $\left(\tau_{H}=2 \pi \hbar / \Delta\right.$, with $\Delta$ the mean level spacing). The matrix $Q$ is an $N \times N$ Hermitian matrix, whose eigenvalues $q_{i}$ 's $(i=1, \ldots, N)$ are the proper delay times measured in units of $\tau_{H}$; that is, $q_{i}=\tau_{i} / \tau_{H}$. The distribution of the proper delay times is known and it is given in terms of their reciprocals. If $x_{i}=\tau_{H} / \tau_{i}$, the joint distribution of the $x_{i}$ 's is given by the Laguerre ensemble, namely 1

$$
P_{\beta}\left(x_{1}, \ldots, x_{N}\right)=C_{N}^{(\beta)} \prod_{a<b}^{N}\left|x_{b}-x_{a}\right|^{\beta} \prod_{c=1}^{N} x_{c}^{\beta N / 2} \mathrm{e}^{-\beta x_{c} / 2},
$$

where $\beta$ characterizes the universal statistics in Dyson's scheme: ${ }^{47} \beta=1$ in the presence of time reversal invariance (TRI) and integral spin or half-integral spin plus rotational symmetry, $\beta=4$ for TRI, half-integral spin and no rotational symmetry, and $\beta=2$ in the absence of TRI. $\frac{5}{5}$ In Eq. (2), $C_{N}^{(\beta)}$ is a normalization constant defined through the condition

$$
\int_{0}^{\infty} P_{\beta}\left(x_{1}, \ldots, x_{N}\right) \mathrm{d} x_{1} \cdots \mathrm{d} x_{N}=1
$$

Generalized moments of the proper delay times (in dimensionless units) like $\left\langle q_{1}^{k_{1}} \cdots q_{N}^{k_{N}}\right\rangle^{(\beta)}$ are just the negative generalized moments of the Laguerre ensemble. That is,

$$
\left\langle q_{1}^{k_{1}} \cdots q_{N}^{k_{N}}\right\rangle^{(\beta)}=\left\langle x_{1}^{-k_{1}} \cdots x_{N}^{-k_{N}}\right\rangle^{(\beta)}=\int_{0}^{\infty} \frac{P_{\beta}\left(x_{1}, \ldots, x_{N}\right)}{x_{1}^{k_{1}} \cdots x_{N}^{k_{N}}} \mathrm{~d} x_{1} \cdots \mathrm{d} x_{N},
$$

for $k_{j}(j=1, \ldots, N)$ an integer number.

\section{B. Partial delay times}

It is instructive to compare the distribution of the proper delay times with the distribution of "partial delay times", defined as the energy derivative of phase-shifts. The distribution of an individual partial delay time scaled with $\tau_{H}$, $\tau_{s}$, for the $\beta=2$ symmetry, is given by ${ }^{37,38}$

$$
P_{s}\left(\tau_{s}\right)=\frac{1}{N !} \tau_{s}^{-N-2} \mathrm{e}^{-1 / \tau_{s}}
$$

In this case it is easy to evaluate the $k$ th moment of the distribution, which is the following:

$$
\left\langle\tau_{s}^{k}\right\rangle=\frac{(N-k) !}{N !}
$$


for $k \leq N$. In particular

$$
\left\langle\tau_{s}\right\rangle=\frac{1}{N} \quad \text { and } \quad\left\langle\tau_{s}^{2}\right\rangle=\frac{1}{(N-1) N},
$$

which is the result expressed in Refs. 36. 37 .

\section{GENERAL EXPRESSIONS FOR THE JOINT MOMENTS}

To obtain a feasible general expression for the joint moments we notice that

$$
\prod_{a<b \leq N}\left(x_{b}-x_{a}\right)=\operatorname{det} V_{N}=\left|\begin{array}{ccccc}
1 & 1 & 1 & \cdots & 1 \\
x_{1} & x_{2} & x_{3} & \cdots & x_{N} \\
\vdots & \vdots & \vdots & \cdots & \vdots \\
x_{1}^{N-1} & x_{2}^{N-1} & x_{3}^{N-1} & \cdots & x_{N}^{N-1}
\end{array}\right|
$$

which is known as the $N$ th-order Vandermonde determinant. 48 It can be proved that ${ }^{49}$

$$
\operatorname{det} V_{N}=\sum_{\sigma} \operatorname{sgn} \sigma \prod_{a=1}^{N} x_{a}^{-1+\sigma(a)}
$$

where $\sigma$ is a permutation that belongs to the symmetric group of degree $N, \underline{50}$

$$
S_{N}=\{\sigma:\{1, \ldots, N\} \rightarrow\{1, \ldots, N\} \mid \sigma \text { is a permutation }\}
$$

with $\operatorname{sgn} \sigma$ the signature of $\sigma$; the identity permutation is $\iota(a)=a$ for $a=1, \ldots, N$.

At this point is necessary to introduce some definitions and properties.

Definition 1. For $t_{a} \geq 0(a=1, \ldots, N)$, let

$$
\Delta_{N}=\left\{\left(t_{1}, \ldots, t_{N}\right): 0 \leq t_{1} \leq t_{2} \leq \cdots \leq t_{N}\right\}
$$

Therefore, the Vandermonde determinant of Eq. (8) becomes positive for $\left(x_{1}, \ldots, x_{N}\right) \in \Delta_{N}$, so that

$$
\operatorname{det} V_{N}=\left|\operatorname{det} V_{N}\right|
$$

and the equality in Eq. (9) can be written as

$$
\left|\operatorname{det} V_{N}\right|^{\beta}=\sum_{\sigma_{1}, \ldots, \sigma_{\beta}} \prod_{i=1}^{\beta} \operatorname{sgn} \sigma_{i} \prod_{a=1}^{N} x_{a}^{-\beta+\sum_{j=1}^{\beta} \sigma_{j}(a)}
$$

where $\sigma_{j} \in S_{N}(j=1, \ldots, \beta)$.

Each permutation $\sigma \in S_{N}$ is associated to an unitary transformation $\pi_{\sigma}$ in the $N$-dimensional Euclidean space:

$$
\left(x_{1}, \ldots, x_{N}\right) \longrightarrow \pi_{\sigma}\left(x_{1}, \ldots, x_{N}\right)=\left[x_{\sigma(1)}, \ldots, x_{\sigma(N)}\right] .
$$

Definition 2. For any $\eta \in S_{N}$ and $t_{a} \geq 0(a=1, \ldots, N)$, let

$$
\Delta_{N}^{\eta}=\pi_{\eta}^{-1}\left(\Delta_{N}\right):=\left\{\left(t_{1}, \ldots, t_{N}\right): 0 \leq t_{\eta(1)} \leq t_{\eta(2)} \leq \cdots \leq t_{\eta(N)}\right\} .
$$

We notice that $\Delta_{N}^{\iota}=\Delta_{N}$; also, for any $\eta \in S_{N}$ and $\left(t_{1}, \ldots, t_{N}\right) \in \Delta_{N}^{\eta}$, from Eq. (14)

$$
\pi_{\eta}^{-1}\left(t_{1}, \ldots, t_{N}\right)=\left[t_{\eta^{-1}(1)}, \ldots, t_{\eta^{-1}(N)}\right] \in \Delta_{N}
$$

and therefore

$$
\operatorname{det} V_{N}\left[\pi_{\eta}^{-1}\left(t_{1}, \ldots, t_{N}\right)\right]=\prod_{a<b}\left[t_{\eta^{-1}(b)}-t_{\eta^{-1}(a)}\right] \geq 0
$$


where Eq. (12) has been taken into account. Hence,

$$
\left|\operatorname{det} V_{N}\left(t_{1}, \ldots, t_{N}\right)\right|=\operatorname{det} V_{N}\left[\pi_{\eta}^{-1}\left(t_{1}, \ldots, t_{N}\right)\right] \text {. }
$$

Finally, for any nonnegative measurable function $f\left(t_{1}, \ldots, t_{N}\right)$, the Change of Variables Theorem allows us to write

$$
\begin{aligned}
\int_{0}^{\infty} f\left(t_{1}, \ldots, t_{N}\right) \mathrm{d} t_{1} \cdots \mathrm{d} t_{N} & =\sum_{\eta \in S_{N}} \int_{\Delta_{N}^{\eta}} f\left(t_{1}, \ldots, t_{N}\right) \mathrm{d} t_{1} \cdots \mathrm{d} t_{N} \\
& =\sum_{\eta \in S_{N}} \int_{\Delta_{N}} f \circ \pi_{\eta}^{-1}\left(t_{1}, \ldots, t_{N}\right) \mathrm{d} t_{1} \cdots \mathrm{d} t_{N}
\end{aligned}
$$

\section{A. Negative moments of the Laguerre distribution of $N$ variables}

From the definition of the Vandermonde determinant (8) and Eq. (13), the Laguerre distribution of Eq. (2) can be written as

$$
P_{\beta}\left(x_{1}, \ldots, x_{N}\right)=C_{N}^{(\beta)} \sum_{\sigma_{1}, \ldots, \sigma_{\beta}} \prod_{i=1}^{\beta} \operatorname{sgn} \sigma_{i} \prod_{a=1}^{N} x_{a}^{\gamma_{a}} \mathrm{e}^{-\beta x_{a} / 2}
$$

where

$$
\gamma_{a}:=\gamma_{a}^{\sigma_{1}, \ldots, \sigma_{\beta}}=\frac{N \beta}{2}-\beta+\sum_{j=1}^{\beta} \sigma_{j}(a) \text { for } a=1, \ldots, N
$$

It is convenient to write $P_{\beta}\left(x_{1}, \ldots, x_{N}\right)$ as

$$
P_{\beta}\left(x_{1}, \ldots, x_{N}\right)=C_{N}^{(\beta)} \sum_{\sigma_{1}, \ldots, \sigma_{\beta}} \prod_{i=1}^{\beta} \operatorname{sgn} \sigma_{i} \prod_{a=1}^{N}\left(\frac{2}{\beta}\right)^{\gamma_{a}+1} \Gamma\left(\gamma_{a}+1\right) f_{\gamma_{a}+1, \beta / 2}\left(x_{a}\right),
$$

where $f_{u, v}(t)$ is the probability density function of the Gamma distribution with parameters $u$ and $v, 51,52$

$$
f_{u, v}(t)=\frac{v^{u} t^{u-1} \mathrm{e}^{-u t}}{\Gamma(u)}
$$

with $\Gamma(u)$ the Gamma function. ${ }^{52}$ It is important to notice that $\Gamma\left(\gamma_{a}+1\right)$ is well defined.

For the joint moments we are interested in, we need the auxiliary function

$$
R_{\beta}\left(x_{1}, \ldots, x_{N}\right)=\frac{P_{\beta}\left(x_{1}, \ldots, x_{N}\right)}{x_{1}^{k_{1}} \cdots x_{N}^{k_{N}}}
$$

for $k_{a}(a=1, \ldots, N)$ an integer number. Using Eq. (22) this function can be written as

$$
R_{\beta}\left(x_{1}, \ldots, x_{N}\right)=C_{N}^{(\beta)} \sum_{\sigma_{1}, \ldots, \sigma_{\beta}} \prod_{i=1}^{\beta} \operatorname{sgn} \sigma_{i} \prod_{a=1}^{N}\left(\frac{2}{\beta}\right)^{\alpha_{a}} \Gamma\left(\alpha_{a}\right) f_{\alpha_{a}, \beta / 2}\left(x_{a}\right),
$$

where $\alpha_{a}=\gamma_{a}-k_{a}+1$ for $a=1, \ldots, N$, with $\gamma_{a}$ as in Eq. (21). Therefore, for any $\eta \in S_{N}$ and $\left(x_{1}, \ldots, x_{N}\right) \in \Delta_{N}^{\eta}$, the properties (16) and (18) allows us to write

$$
R_{\beta} \circ \pi_{\eta}^{-1}\left(x_{1}, \ldots, x_{N}\right)=\frac{P_{\beta}\left(x_{1}, \ldots, x_{N}\right)}{x_{\eta^{-1}(1)}^{k_{1}} \cdots x_{\eta^{-1}(N)}^{k_{N}}}=\frac{P_{\beta}\left(x_{1}, \ldots, x_{N}\right)}{x_{1}^{k_{\eta(1)}} \cdots x_{N}^{k_{\eta(N)}}} .
$$

The last equality is just the definition (24), which according to Eq. (25), it can be written as

$$
R_{\beta} \circ \pi_{\eta}^{-1}\left(x_{1}, \ldots, x_{N}\right)=C_{N}^{(\beta)} \sum_{\sigma_{1}, \ldots, \sigma_{\beta}} \prod_{i=1}^{\beta} \operatorname{sgn} \sigma_{i} \prod_{a=1}^{N}\left(\frac{2}{\beta}\right)^{\alpha_{a}^{\eta}} \Gamma\left(\alpha_{a}^{\eta}\right) f_{\alpha_{a}^{\eta}, \beta / 2}\left(x_{a}\right)
$$


where

$$
\alpha_{a}^{\eta}=\gamma_{a}-k_{\eta(a)}+1, \quad \text { for } \quad a=1, \ldots, N
$$

If we integrate this equation over $\Delta_{N}$ and sum over $\eta$, the property (19) allows us to arrive to the desired result, namely

$$
\left\langle x_{1}^{-k_{1}} \cdots x_{N}^{-k_{N}}\right\rangle^{(\beta)}=C_{N}^{(\beta)} \sum_{\eta, \sigma_{1}, \ldots, \sigma_{\beta}} F_{\theta_{\eta, N}} \prod_{i=1}^{\beta} \operatorname{sgn} \sigma_{i} \prod_{a=1}^{N}\left(\frac{2}{\beta}\right)^{\alpha_{a}^{\eta}} \Gamma\left(\alpha_{a}^{\eta}\right) .
$$

where $F_{\theta_{\eta, N}}$ is defined as

$$
F_{\theta_{\eta, N}}=\int_{\Delta_{N}} \prod_{a=1}^{N} f_{\alpha_{a}^{\eta}, \beta / 2}\left(x_{a}\right) \mathrm{d} x_{a}
$$

with

$$
\theta_{\eta, N}:=\theta_{\eta, N}^{\sigma_{1}, \ldots, \sigma_{\beta}}:=(\alpha_{1}^{\eta}, \ldots, \alpha_{N}^{\eta}, \underbrace{\beta / 2, \ldots, \beta / 2}_{N}) .
$$

It is clear that the normalization constant $C_{N}^{(\beta)}$ can be obtained from Eq. (29) for $k_{a}=0(a=1, \ldots, N)$, in which case the argument in the sum of Eq. (29) is independent of $\eta$, such that the sum over $\eta$ is exactly $N$ !. It is given by

$$
C_{N}^{(\beta)}=\left[N ! \sum_{\sigma_{1}, \ldots, \sigma_{\beta}} F_{\theta_{N}} \prod_{i=1}^{\beta} \operatorname{sgn} \sigma_{i} \prod_{a=1}^{N}\left(\frac{2}{\beta}\right)^{\gamma_{a}+1} \Gamma\left(\gamma_{a}+1\right)\right]^{-1},
$$

where

$$
F_{\theta_{N}}=\int_{\Delta_{N}} \prod_{a=1}^{N} f_{\gamma_{a}+1, \beta / 2}\left(x_{a}\right) \mathrm{d} x_{a}
$$

Two Remarks are worth mentioning.

Remark 1. In Eqs. (29) and (32), it is necessary to take into account the dependence of the parameters $\theta_{N}$ and $\theta_{\eta, N}$ on the permutations $\sigma_{1}, \ldots, \sigma_{\beta}$ but we omitted to write it explicitly, for simplicity.

Remark 2. Since the minimum possible value of any permutation is 1 , it is easy to show that $\gamma_{a}-k_{a}+1>0$ for $0 \leq k_{a}<\frac{N \beta}{2}+1$.

This Remark ensures that the Gamma function that appears in Eq. (29) is well defined. It is important to notice that the property expressed in Remark 2 is a general result that restricts the values of $k_{a}(a=1, \ldots, N)$. This condition is the most general and coincides with that of Ref. 45 for the particular case when $k_{a}=k$, for $a=1, \ldots, N$. The restriction of $k$ in Eq. (6) appears also as a particular case.

\section{1. $\beta$ an even integer number}

When $\beta$ is an even positive integer then $\left|\operatorname{det} V_{N}\right|^{\beta}=\left(\operatorname{det} V_{N}\right)^{\beta}$ for any $\left(x_{1}, \ldots, x_{N}\right) \in \mathbb{R}^{N}$, being $\mathbb{R}^{N}$ the real $N$-dimensional Euclidean space. In this case there is no need to use Eq. (19) so, in the computations of the negative moments, we obtain simpler formulas for the joint negative moments of the delay times.

If we integrate Eq. (25) we have that

$$
\begin{aligned}
\left\langle x_{1}^{-k_{1}} \cdots x_{N}^{-k_{N}}\right\rangle^{(\beta)} & =C_{N}^{(\beta)}\left(\frac{2}{\beta}\right)^{N} \sum_{\sigma_{1}, \ldots, \sigma_{\beta}} \prod_{i=1}^{\beta} \operatorname{sgn} \sigma_{i} \prod_{a=1}^{N} \int_{0}^{\infty}\left(\frac{\beta}{2}\right) x^{\gamma_{a}-k_{a}} \mathrm{e}^{-\beta x / 2} \mathrm{~d} x \\
& =C_{N}^{(\beta)}\left(\frac{2}{\beta}\right)^{N} A_{N}^{(\beta)}\left(k_{1}, \ldots, k_{N}\right),
\end{aligned}
$$

where

$$
A_{N}^{(\beta)}\left(k_{1}, \ldots, k_{N}\right)=\sum_{\sigma_{1}, \ldots, \sigma_{\beta}} \prod_{i=1}^{\beta} \operatorname{sgn} \sigma_{i} \prod_{a=1}^{N}\left(\frac{2}{\beta}\right)^{\gamma_{a}-k_{a}} \Gamma\left(\gamma_{a}-k_{a}+1\right)
$$

If $k_{1}=\cdots=k_{N}=0$, then Eq. (34) implies that $C_{N}^{(\beta)}=(\beta / 2)^{N} / A_{N}^{(\beta)}$, with $A_{N}^{(\beta)}=A_{N}^{(\beta)}(0, \ldots, 0)$. 


\section{EXPLICIT COMPUTATIONS}

We present explicit calculations with $N=1$ and 2 , for $\beta=1$ and 4 , and $N=1,2,3$ and 4 for $\beta=2$. We make explicit, as far as possible, the dependence on the corresponding permutations. We start our calculations with the $\beta=1$ case since it is usually the most difficult to treat analytically.

\section{A. Explicit calculations for $\beta=1$}

For the particular case of $\beta=1$ but arbitrary $N$, Eq. (21) is written as

$$
\gamma_{a}:=\gamma_{a}^{\sigma_{1}}=\frac{N}{2}-1+\sigma_{1}(a), \quad \text { for } \quad a=1, \ldots, N
$$

such that the moments given by Eqs. (29) are simplified to

$$
\left\langle x_{1}^{-k_{1}} \cdots x_{N}^{-k_{N}}\right\rangle^{(1)}=C_{N}^{(1)} \sum_{\eta, \sigma_{1}} F_{\theta_{\eta, N}} \operatorname{sgn} \sigma_{1} \prod_{a=1}^{N} 2^{\sigma_{1}(a)-k_{\eta(a)}+N / 2} \Gamma\left[\sigma_{1}(a)-k_{\eta(a)}+N / 2\right],
$$

where $0 \leq k_{a}<1+N / 2$.

$$
\text { 1. The } N=1 \text { case }
$$

According to Eq. (10), $S_{1}=\{\iota(1)=1\}$, that is, only the identity belongs to the symmetric group $S_{1}$, such that $\eta(1)=\sigma_{1}(1)=\iota(1)=1$ and $\theta_{\eta, 1}=\theta_{1}=(3 / 2-k, 1 / 2)$. Therefore, from Eq. (37),

$$
\left\langle x^{-k}\right\rangle^{(1)}=C_{1}^{(1)} F_{\theta_{1}} 2^{-k+3 / 2} \Gamma(-k+3 / 2)=C_{1}^{(1)} 2^{-k+3 / 2} \Gamma(-k+3 / 2)
$$

where we have used that

$$
F_{\theta_{1}}=\frac{1}{2^{3 / 2} \Gamma(3 / 2)} \int_{0}^{\infty} t^{1 / 2} \mathrm{e}^{-t} \mathrm{~d} t=1
$$

The only values that $k$ can take are 0 and 1 . For $k=0$ we obtain the normalization constant: $C_{1}^{(1)}=1 / 2^{3 / 2}(1 / 2)$ !, while for $k=1$ we have

$$
\left\langle x_{1}^{-k}\right\rangle^{(1)}=\left(\frac{1}{2}\right)^{k} \frac{\left(\frac{1}{2}-k\right) !}{\left(\frac{1}{2}\right) !} .
$$

$$
\text { 2. The } N=2 \text { case }
$$

In this case, $S_{2}=\{\iota, \sigma\}$, such that $\sigma_{1}=\iota, \sigma$ with $\iota(a)=a(a=1,2), \sigma(1)=2$ and $\sigma(2)=1$; therefore, $\gamma_{a}=\sigma_{1}(a)$. Equation Eq. (37) for $N=2$ gives

$$
\left\langle x_{1}^{-k_{1}} x_{2}^{-k_{2}}\right\rangle^{(1)}=C_{2}^{(1)} 2^{5-k_{1}-k_{2}}\left[\left(F_{\theta_{\iota, 2}^{\iota}}-F_{\theta_{\sigma, 2}^{\sigma}}\right) \Gamma\left(2-k_{1}\right) \Gamma\left(3-k_{2}\right)+\left(F_{\theta_{\sigma, 2}^{\iota}}-F_{\theta_{\iota, 2}^{\sigma}}\right) \Gamma\left(3-k_{1}\right) \Gamma\left(2-k_{2}\right)\right] .
$$

Here, Eq. (28) says that $\alpha_{a}^{\eta}=\sigma_{1}(a)-k_{\eta(a)}+1$ and Eq. (31) gives

$$
\theta_{\eta, N}^{\sigma_{1}}=\left[\sigma_{1}(i)-k_{\eta(1)}+1, \sigma_{1}(2)-k_{\eta(2)}+1,1 / 2,1 / 2\right] .
$$

According to Eq. (A12) of the Appendix , we can determine the coefficients $F_{\theta_{\eta, N}}^{\sigma_{1}}$ by means of the negative binomial distribution $N B_{\alpha_{2}, p_{2}}$ with parameters $\alpha_{2}$ and $p_{2}=1 / 2 \underline{\underline{53}}$ For the particular case of $N=2$

$$
F_{\theta_{\eta, 2}}^{\sigma_{1}}=1-\sum_{\ell=0}^{\sigma_{1}(1)-k_{\eta(1)}} N B_{\sigma_{1}(2)-k_{\eta(2)}+1,1 / 2}(\ell)
$$


for any $\eta$ and $\sigma_{1} \in S_{2}$ and $k_{a}=0,1$.

For the particular case of $k_{1}=k_{2}=0$ we have that

$$
\begin{aligned}
& F_{\theta_{2}^{\iota}}=1-\sum_{\ell=0}^{\iota(1)} N B_{\iota(2)+1,1 / 2}(\ell)=1-\sum_{\ell=0}^{1} N B_{3,1 / 2}(\ell)=\frac{11}{16}, \\
& F_{\theta_{2}^{\sigma}}=1-\sum_{\ell=0}^{\sigma(1)} N B_{\sigma(2)+1,1 / 2}(\ell)=1-\sum_{\ell=0}^{2} N B_{2,1 / 2}(\ell)=\frac{5}{16},
\end{aligned}
$$

where we have used the Definition (A4), and the normalization constant is $C_{2}^{(1)}=1 / 48$.

In very similar way, for $k_{1}=k=1$ and $k_{2}=0$,

$$
F_{\theta_{\iota, 2}^{\iota}}=\frac{7}{8}, \quad F_{\theta_{\sigma, 2}^{\iota}}=\frac{1}{2}, \quad F_{\theta_{\iota, 2}^{\sigma}}=\frac{1}{2}, \quad \text { and } \quad F_{\theta_{\sigma, 2}^{\sigma}}=\frac{1}{8}
$$

such that

$$
\left\langle x_{1}^{-k}\right\rangle^{(1)}=\left(\frac{1}{2}\right)^{k} \frac{(1-k) !}{1 !} K_{2}^{(1)}(k, 0)
$$

where

$$
K_{2}^{(1)}(k, 0)=1 \quad \text { for } \quad k=1
$$

For $k_{1}=k_{2}=k=1$ we have that

$$
F_{\theta_{\iota, 2}^{\iota}}=\frac{3}{4}, \quad F_{\theta_{\sigma, 2}^{\iota}}=\frac{3}{4}, \quad F_{\theta_{\iota, 2}^{\sigma}}=\frac{1}{4}, \quad \text { and } \quad F_{\theta_{\sigma, 2}^{\sigma}}=\frac{1}{4},
$$

and we can write the corresponding moment as

$$
\left\langle x_{1}^{-k} x_{2}^{-k}\right\rangle^{(1)}=\left(\frac{1}{2}\right)^{2 k} \frac{(1-k) !}{1 !} \frac{\left(\frac{3}{2}-k\right) !}{\frac{3}{2} !} .
$$

For $\beta=1$, the calculations become much more complicated for values of $N$ larger than 2 . However, some moments can be obtained numerically, some of which are shown in Section IVD

\section{B. Explicit calculations for $\beta=2$}

From Eq. (21) we have that $\gamma_{a}=N-2+\sigma_{1}(a)+\sigma_{2}(a)(a=1, \ldots, N)$ and Eq. (35) is written as

$$
A_{N}^{(2)}\left(k_{1}, \ldots, k_{N}\right)=\sum_{\sigma_{1}, \sigma_{2} \in S_{N}} \operatorname{sgn}\left(\sigma_{1} \sigma_{2}\right) \prod_{a=1}^{N}\left[N-2+\sigma_{1}(a)+\sigma_{2}(a)-k_{a}\right] ! ;
$$

the normalization constant is $C_{N}^{(2)}=1 / A_{N}^{(2)}$, with $A_{N}^{(2)}=A_{N}^{(2)}(0, \ldots, 0)$, and the moments are given by Eq. (34).

$$
\text { 1. The } N=1 \text { case }
$$

In this special case, $\gamma_{1}=\sigma_{1}+\sigma_{2}-1$, such that Eq. (51) reads

$$
A_{1}^{(2)}(k)=\sum_{\sigma_{1}, \sigma_{2} \in S_{1}} \operatorname{sgn}\left(\sigma_{1} \sigma_{2}\right)\left(\sigma_{1}+\sigma_{2}-k-1\right) !=(1-k) ! .
$$

Since $N \beta / 2+1=2$, according to Remark 2 the maximum value for $k$ is 1 . The normalization constant is obtained for $k=0$ as $C_{1}^{(2)}=1 / 1$ !. Therefore, the only moment is

$$
\left\langle x_{1}^{-k}\right\rangle^{(2)}=\frac{(1-k) !}{1 !} \text { for } \quad k=1 .
$$




\section{The $N=2$ case}

In this case, we observe that $\gamma_{a}=\sigma_{1}(a)+\sigma_{2}(a)(a=1,2)$ and Eq. (51) becomes

$$
A_{2}^{(2)}\left(k_{1}, k_{2}\right)=\left(2-k_{1}\right) !\left(4-k_{2}\right) !+\left(4-k_{1}\right) !\left(2-k_{2}\right) !-2\left(3-k_{1}\right) !\left(3-k_{2}\right) !
$$

Here, $N \beta / 2+1=3$ such that the maximum order of the negative moments is 2 ; that is, $k_{a}=0,1,2(a=1,2)$.

For $k_{1}=k_{2}=0$ we obtain the normalization constant, $C_{2}^{(2)}=1 / 2$ ! (3! $\cdot 2 ! \cdot 1$ !). The corresponding moments for $k_{1}=k$ and $k_{2}=0$ are

$$
\left\langle x_{1}^{-k}\right\rangle^{(2)}=\frac{(2-k) !}{2 !} K_{2}^{(2)}(k, 0)
$$

where

$$
K_{2}^{(2)}(k, 0)=\frac{1}{3 !}[12-6(3-k)+(3-k)(4-k)]
$$

Also, for $k_{1}=k_{2}=k$ we have

$$
\left\langle x_{1}^{-k} x_{2}^{-k}\right\rangle^{(2)}=\frac{(3-k) !}{3 !} \frac{(2-k) !}{2 !} .
$$

The remaining joint negative moment is

$$
\left\langle x_{1}^{-2} x_{2}^{-1}\right\rangle^{(2)}=\frac{1 !}{3 !}
$$

$$
\text { 3. The } N=3 \text { case }
$$

For $N=3, \gamma_{a}=\sigma_{1}(a)+\sigma_{2}(a)+1(a=1,2,3)$ and Eq. (151) becomes

$$
\begin{aligned}
A_{3}^{(2)}\left(k_{1}, k_{2}, k_{3}\right) & =\left(3-k_{1}\right) !\left(5-k_{2}\right) !\left(7-k_{3}\right) !+\left(3-k_{1}\right) !\left(7-k_{2}\right) !\left(5-k_{3}\right) ! \\
& +\left(5-k_{1}\right) !\left(3-k_{2}\right) !\left(7-k_{3}\right) !+\left(5-k_{1}\right) !\left(7-k_{2}\right) !\left(3-k_{3}\right) ! \\
& +\left(7-k_{1}\right) !\left(3-k_{2}\right) !\left(5-k_{3}\right) !+\left(7-k_{1}\right) !\left(5-k_{2}\right) !\left(3-k_{3}\right) ! \\
& -2\left(3-k_{1}\right) !\left(6-k_{2}\right) !\left(6-k_{3}\right) !-2\left(6-k_{1}\right) !\left(3-k_{2}\right) !\left(6-k_{3}\right) ! \\
& -2\left(6-k_{1}\right) !\left(6-k_{2}\right) !\left(3-k_{3}\right) !-2\left(4-k_{1}\right) !\left(4-k_{2}\right) !\left(7-k_{3}\right) ! \\
& -2\left(4-k_{1}\right) !\left(7-k_{2}\right) !\left(4-k_{3}\right) !-2\left(7-k_{1}\right) !\left(4-k_{2}\right) !\left(4-k_{3}\right) ! \\
& +2\left(4-k_{1}\right) !\left(5-k_{2}\right) !\left(6-k_{3}\right) !+2\left(4-k_{1}\right) !\left(6-k_{2}\right) !\left(5-k_{3}\right) ! \\
& +2\left(6-k_{1}\right) !\left(5-k_{2}\right) !\left(4-k_{3}\right) !+2\left(5-k_{1}\right) !\left(4-k_{2}\right) !\left(6-k_{3}\right) ! \\
& +2\left(5-k_{1}\right) !\left(6-k_{2}\right) !\left(4-k_{3}\right) !+2\left(6-k_{1}\right) !\left(4-k_{2}\right) !\left(5-k_{3}\right) ! \\
& -6\left(5-k_{1}\right) !\left(5-k_{2}\right) !\left(5-k_{3}\right) !
\end{aligned}
$$

The maximum value of $k_{a}(a=1,2,3)$ is 3 . If we evaluate this expression at $k_{1}=k_{2}=k_{3}=0$ we obtain $C_{3}^{(2)}=1 / 3 !(5 ! \cdot 4 ! \cdot 3 ! \cdot 2 ! \cdot 1 !)$.

The result for the moments for $k_{1}=k$ and $k_{2}=k_{3}=0$ is given by

$$
\left\langle x_{1}^{-k}\right\rangle^{(2)}=\frac{(3-k) !}{3 !} K_{3}^{(2)}(k, 0,0),
$$

where

$$
\begin{aligned}
K_{3}^{(2)}(k, 0,0) & =\frac{1}{6 !}[5 \cdot 6 !-4 \cdot 6 !(4-k)+150 \cdot 3 !(4-k)(5-k) \\
& -5 !(4-k)(5-k)(6-k)+3 !(4-k)(5-k)(6-k)(7-k)]
\end{aligned}
$$

In similar way, for $k_{1}=k_{2}=k$ and $k_{3}=0$ we have that

$$
\left\langle x_{1}^{-k} x_{2}^{-k}\right\rangle^{(2)}=\frac{(3-k) !}{3 !} \frac{(4-k) !}{4 !} K_{3}^{(2)}(k, k, 0),
$$


where

$$
\begin{aligned}
K_{3}^{(2)}(k, k, 0) & =\frac{1}{6 !}[7 !-6 !(k-1)(5-k) \\
& \left.-4 \cdot 4 !(4-k)(5-k)(6-k)+3 !(4-k)(5-k)^{2}(6-k)\right] .
\end{aligned}
$$

The moments for $k_{1}=k_{2}=k_{3}=k$ are given by

$$
\left\langle x_{1}^{-k} x_{2}^{-k} x_{3}^{-k}\right\rangle^{(2)}=\frac{(5-k) !}{5 !} \frac{(4-k) !}{4 !} \frac{(3-k) !}{3 !} .
$$

The remaining terms can be evaluated directly from Eq. (59). They are the following:

$$
\left\langle x_{1}^{-2} x_{2}^{-1}\right\rangle^{(2)}=\frac{3 !}{5 !}, \quad\left\langle x_{1}^{-3} x_{2}^{-1}\right\rangle^{(2)}=\frac{62}{6 !}, \quad \text { and } \quad\left\langle x_{1}^{-3} x_{2}^{-2}\right\rangle^{(2)}=\frac{3}{5 !} ;
$$

also,

$$
\begin{gathered}
\left\langle x_{1}^{-2} x_{2}^{-1} x_{3}^{-1}\right\rangle^{(2)}=\frac{6}{6 !},\left\langle x_{1}^{-2} x_{2}^{-2} x_{3}^{-1}\right\rangle^{(2)}=\frac{2 !}{6 !},\left\langle x_{1}^{-3} x_{2}^{-1} x_{3}^{-1}\right\rangle^{(2)}=\frac{10}{6 !},\left\langle x_{1}^{-3} x_{2}^{-2} x_{3}^{-1}\right\rangle^{(2)}=\frac{5}{2 \cdot 6 !} ; \\
\left\langle x_{1}^{-3} x_{2}^{-2} x_{3}^{-2}\right\rangle^{(2)}=\frac{1}{2 \cdot 6 !}, \quad\left\langle x_{1}^{-3} x_{2}^{-3} x_{3}^{-1}\right\rangle^{(2)}=\frac{1}{4 \cdot 5 !}, \quad \text { and } \quad\left\langle x_{1}^{-3} x_{2}^{-3} x_{3}^{-2}\right\rangle^{(2)}=\frac{1}{4 \cdot 6 !} .
\end{gathered}
$$

$$
\text { 4. The } N=4 \text { case }
$$

From Eq. (21), $\gamma_{a}=\sigma_{1}(a)+\sigma_{2}(a)+2(a=1,2,3,4)$; in this case Eq (51) gives

$$
\begin{aligned}
A_{4}^{(2)}\left(k_{1}, k_{2}, k_{3}, k_{4}\right) & =\left(4-k_{1}\right) !\left(6-k_{2}\right) !\left(8-k_{3}\right) !\left(10-k_{4}\right) !-\left(4-k_{1}\right) !\left(6-k_{2}\right) !\left(9-k_{3}\right) !\left(9-k_{4}\right) ! \\
& -\left(4-k_{1}\right) !\left(7-k_{2}\right) !\left(7-k_{3}\right) !\left(10-k_{4}\right) !+\left(4-k_{1}\right) !\left(7-k_{2}\right) !\left(9-k_{3}\right) !\left(8-k_{4}\right) ! \\
& +\left(4-k_{1}\right) !\left(8-k_{2}\right) !\left(7-k_{3}\right) !\left(9-k_{4}\right) !-\left(4-k_{1}\right) !\left(8-k_{2}\right) !\left(8-k_{3}\right) !\left(8-k_{4}\right) ! \\
& -\left(5-k_{1}\right) !\left(5-k_{2}\right) !\left(8-k_{3}\right) !\left(10-k_{4}\right) !+\left(5-k_{1}\right) !\left(5-k_{2}\right) !\left(9-k_{3}\right) !\left(9-k_{4}\right) ! \\
& +\left(5-k_{1}\right) !\left(7-k_{2}\right) !\left(6-k_{3}\right) !\left(10-k_{4}\right) !-\left(5-k_{1}\right) !\left(7-k_{2}\right) !\left(9-k_{3}\right) !\left(7-k_{4}\right) ! \\
& -\left(5-k_{1}\right) !\left(8-k_{2}\right) !\left(6-k_{3}\right) !\left(9-k_{4}\right) !+\left(5-k_{1}\right) !\left(8-k_{2}\right) !\left(8-k_{3}\right) !\left(7-k_{4}\right) ! \\
& +\left(6-k_{1}\right) !\left(5-k_{2}\right) !\left(7-k_{3}\right) !\left(10-k_{4}\right) !-\left(6-k_{1}\right) !\left(5-k_{2}\right) !\left(9-k_{3}\right) !\left(8-k_{4}\right) ! \\
& -\left(6-k_{1}\right) !\left(6-k_{2}\right) !\left(6-k_{3}\right) !\left(10-k_{4}\right) !+\left(6-k_{1}\right) !\left(6-k_{2}\right) !\left(9-k_{3}\right) !\left(7-k_{4}\right) ! \\
& +\left(6-k_{1}\right) !\left(8-k_{2}\right) !\left(6-k_{3}\right) !\left(8-k_{4}\right) !-\left(6-k_{1}\right) !\left(8-k_{2}\right) !\left(7-k_{3}\right) !\left(7-k_{4}\right) ! \\
& -\left(7-k_{1}\right) !\left(5-k_{2}\right) !\left(7-k_{3}\right) !\left(9-k_{4}\right) !+\left(7-k_{1}\right) !\left(5-k_{2}\right) !\left(8-k_{3}\right) !\left(8-k_{4}\right) ! \\
& +\left(7-k_{1}\right) !\left(6-k_{2}\right) !\left(6-k_{3}\right) !\left(9-k_{4}\right) !-\left(7-k_{1}\right) !\left(6-k_{2}\right) !\left(8-k_{3}\right) !\left(7-k_{4}\right) ! \\
& -\left(7-k_{1}\right) !\left(7-k_{2}\right) !\left(6-k_{3}\right) !\left(8-k_{4}\right) !+\left(7-k_{1}\right) !\left(7-k_{2}\right) !\left(7-k_{3}\right) !\left(7-k_{4}\right) ! \\
& +\left(\text { permutations of } k_{1}, k_{2}, k_{3}, k_{4}\right) .
\end{aligned}
$$

This equation is well defined because the maximum allowed value for $k_{a}(a=1,2,3,4)$ is 4 .

Although the calculation of $A_{4}^{(2)}\left(k_{1}, k_{2}, k_{3}, k_{4}\right)$ for arbitrary set of values of the $k_{a}$ 's is not difficult, it consists of many terms that are not easy to follow. Two quantities are clearly feasible: one for $k_{a}=0$ and the other for $k_{a}=k$. This is due to the fact that the permutations of $k_{a}$ 's in Eq. (68) give the same terms that have been explicitly written. The first quantity gives the normalization constant, $C_{4}^{(2)}=1 / 4 !(7 ! \cdot 6 ! \cdot 5 ! \cdot 4 ! \cdot 3 ! \cdot 2 ! \cdot 1 !)$. The second quantity is the moment

$$
\left\langle x_{1}^{-k} x_{2}^{-k} x_{3}^{-k} x_{4}^{-k}\right\rangle^{(2)}=\frac{(7-k) !}{7 !} \frac{(6-k) !}{6 !} \frac{(5-k) !}{5 !} \frac{(4-k) !}{4 !} .
$$

Any other moment is difficult to compute arithmetically, with great effort we arrive to the following results:

$$
\left\langle x_{1}^{-1}\right\rangle^{(2)}=\frac{3 !}{4 !}, \quad\left\langle x_{1}^{-2}\right\rangle^{(2)}=\frac{8 \cdot 2 !}{5 !}, \quad\left\langle x_{1}^{-3}\right\rangle^{(2)}=\frac{16 \cdot 3 !}{6 !}, \quad \text { and } \quad\left\langle x_{1}^{-4}\right\rangle^{(2)}=\frac{59 \cdot 4 !}{7 !} ;
$$




$$
\begin{aligned}
& \left\langle x_{1}^{-1} x_{2}^{-1}\right\rangle^{(2)}=\frac{6}{5 !}, \quad\left\langle x_{1}^{-2} x_{2}^{-2}\right\rangle^{(2)}=\frac{3}{6 !}\left(\frac{8 \cdot 9}{6 \cdot 7}\right), \quad \text { and } \quad\left\langle x_{1}^{-3} x_{2}^{-3}\right\rangle^{(2)}=\frac{8 \cdot 5 !}{9 !} \\
& \left\langle x_{1}^{-1} x_{2}^{-1} x_{3}^{-1}\right\rangle^{(2)}=\frac{1}{5 !}, \quad\left\langle x_{1}^{-2} x_{2}^{-2} x_{3}^{-2}\right\rangle^{(2)}=\frac{1}{7 !}, \quad \text { and } \quad\left\langle x_{1}^{-3} x_{2}^{-3} x_{3}^{-3}\right\rangle^{(2)}=\frac{51}{10 !} .
\end{aligned}
$$

\section{Explicit calculations for $\beta=4$}

For $\beta=4$, Eq. (21) says that $\gamma_{a}=2 N-4+\sigma_{1}(a)+\sigma_{2}(a)+\sigma_{3}(a)+\sigma_{4}(a)(a=1, \ldots, N)$, such that Eq. (35) gives

$$
A_{N}^{(4)}\left(k_{1}, \ldots, k_{N}\right)=\sum_{\sigma_{1}, \sigma_{2}, \sigma_{3} \sigma_{4} \in S_{N}} \operatorname{sgn}\left(\sigma_{1} \sigma_{2} \sigma_{3} \sigma_{4}\right) \prod_{i=1}^{N} \frac{\left(\gamma_{i}-k_{i}\right) !}{2^{\gamma_{i}-k_{i}}} .
$$

The normalization constant is given by $C_{N}^{(4)}=2^{N} / A_{N}^{(4)}$ and the moments are given by Eq. (34).

$$
\text { 1. The } N=1 \text { case }
$$

In this case, $\gamma=\left(\sigma_{1}+\sigma_{2}+\sigma_{3}+\sigma_{4}-k-2\right)$ !. Equation (73) can be written as

$$
A_{1}^{(4)}(k)=2^{k} \frac{(2-k) !}{2^{2}},
$$

such that $A_{1}^{(4)}=1 / 2$ and $C_{1}^{(4)}=2^{2}$. Therefore,

$$
\left\langle x_{1}^{-k}\right\rangle^{(4)}=2^{k} \frac{(2-k) !}{2 !} \quad \text { for } \quad k=1,2 .
$$

$$
\text { 2. The } N=2 \text { case }
$$

Here, $\sigma_{i} \in S_{2}(i=1,2,3,4)$ and

$$
\begin{aligned}
A_{2}^{(4)}\left(k_{1}, k_{2}\right) & =2^{k_{1}+k_{2}-12}\left[\left(4-k_{1}\right) !\left(8-k_{2}\right) !-4\left(5-k_{1}\right) !\left(7-k_{2}\right) !+3\left(6-k_{1}\right) !\left(6-k_{2}\right) !\right] \\
& +\left(\text { permutations of } k_{1} \text { and } k_{2}\right) .
\end{aligned}
$$

Taking $k_{1}=k_{2}=0$, we have that $C_{2}^{(4)}=2^{14} / 3 !(5 ! \cdot 4 ! \cdot 3 ! \cdot 2 ! \cdot 1 !)$, while for $k_{1}=k_{2}=k$,

$$
\left\langle x_{1}^{-k} x_{2}^{-k}\right\rangle^{(4)}=2^{2 k} \frac{(6-k) !}{6 !} \frac{(4-k) !}{4 !}, \quad \text { for } \quad k=1,2,3,4 .
$$

Also, for $k_{1}=k$ and $k_{2}=0$, we get

$$
\left\langle x_{1}^{-k}\right\rangle^{(4)}=2^{k} \frac{(4-k) !}{4 !} K_{2}^{(4)}(k, 0),
$$

where

$$
K_{2}^{(4)}(k, 0)=\frac{1}{6 ! \cdot 3 ! \cdot 2 !}[8 !+2 \cdot 6 !(5-k)(4-3 k)-4 !(12+k)(7-k)(6-k)(5-k)] .
$$

From Eq. (76) it is easy to see that the remaining moments are given by

$$
\begin{aligned}
& \left\langle x_{1}^{-2} x_{2}^{-1}\right\rangle^{(4)}=\frac{1}{9}, \quad\left\langle x_{1}^{-3} x_{2}^{-1}\right\rangle^{(4)}=\frac{7}{45}, \quad \text { and } \quad\left\langle x_{1}^{-3} x_{2}^{-2}\right\rangle^{(4)}=\frac{2}{45} \\
& \left\langle x_{1}^{-4} x_{2}^{-1}\right\rangle^{(4)}=\frac{22}{45}, \quad\left\langle x_{1}^{-4} x_{2}^{-2}\right\rangle^{(4)}=\frac{2}{15}, \quad \text { and } \quad\left\langle x_{1}^{-4} x_{2}^{-3}\right\rangle^{(4)}=\frac{2}{45} .
\end{aligned}
$$




\section{Joint moments of proper delay times for arbitraries $N$ and $\beta$}

The set of equations (40), (47), (53), (55), (60), (75), and (78); (150), (57), (62), and (77); (64) and (69), suggest a general expression for the joint moments for any symmetry and number of channels, which is

$$
\left\langle q_{1}^{k} \cdots q_{m}^{k}\right\rangle^{(\beta)}=\left[\prod_{n=N}^{N+m-1}\left(\frac{\beta}{2}\right)^{k} \frac{\left(\frac{\beta n}{2}-k\right) !}{\left(\frac{\beta n}{2}\right) !}\right] K_{N}^{(\beta)}(\underbrace{k, \ldots, k}_{m}, \underbrace{0, \ldots, 0}_{N-m}) \text { for } m \leq N,
$$

where $K_{N}^{(\beta)}(\underbrace{k, \ldots, k}_{m}, \underbrace{0, \ldots, 0}_{N-m})$ has a particular expression for each values of $N$ and $m$, as can be seen in Eqs. (48), (56), (61), (63), and (79). A closed expression for this quantity is difficult to obtain analytically, but it reduces to 1 for $m=N$, as is suggested also by Eqs. (40), (50), (53), (57), (64), (69), (75), and (77),

$$
K_{N}^{(\beta)}(k, \ldots, k)=1
$$

as well as for $k=1$ and $m \leq N$,

$$
K_{N}^{(\beta)}(\underbrace{1, \ldots, 1}_{m}, \underbrace{0, \ldots, 0}_{N-m})=1
$$

as can be seen by the direct evaluation of the equations just mentioned above.

A particular case of interest is the $k$ th moment for $m=1$ (single variable); we find that

$$
\left\langle q_{1}^{k}\right\rangle^{(\beta)}=\left(\frac{\beta}{2}\right)^{k} \frac{\left(\frac{\beta N}{2}-k\right) !}{\left(\frac{\beta N}{2}\right) !} K_{N}^{(\beta)}(k, 0, \ldots, 0), \quad \text { for } \quad k<\frac{\beta N}{2}+1 .
$$

What is interesting of this result is that the $k$ th moment of a proper delay time differs from that of a partial delay time, as can be seen if we compare this result for $\beta=2$ with Eq. (6) 36,37 Moreover, it allows us to generalize the $k$ th moment for a partial time for any $\beta$ and $N$, namely

$$
\left\langle\tau_{s}^{k}\right\rangle^{(\beta)}=\left(\frac{\beta}{2}\right)^{k} \frac{\left(\frac{\beta N}{2}-k\right) !}{\left(\frac{\beta N}{2}\right) !} \text { for } \quad k<\frac{\beta N}{2}+1
$$

That is, the distribution for the partial times can be also generalized to any $\beta$ and $N$ by replacing $N$ by $\beta N / 2$ in the corresponding distribution of the $\beta=2$ case of Eq. (5) ${ }^{36}-38$ The quantitative difference given by the factor $K_{N}^{(\beta)}(k, 0, \ldots, 0)$ in Eq. (85), comes from the level repulsion for the proper delay times, as happens for their corresponding density. ${ }^{2}$ The only exception to this rule is, of course, the case $N=1$.

We can also notice that the factor $K_{N}^{(\beta)}(k, 0, \ldots, 0)$ has the following expression:

$$
K_{N}^{(\beta)}(k, 0, \ldots, 0)=\frac{k ! N^{k-1} N !}{(N+k-1) !} \quad \text { for } \quad k=1,2,3
$$

independent of $\beta$.

From Eqs. (85) and (87) we obtain the following interesting averages:

$$
\left\langle q_{1}\right\rangle^{(\beta)}=\frac{1}{N}, \quad\left\langle q_{1}^{2}\right\rangle^{(\beta)}=\frac{\beta}{2} \frac{2}{\left(\frac{\beta N}{2}-1\right)(N+1)},
$$

and

$$
\left\langle q_{1}^{3}\right\rangle^{(\beta)}=\left(\frac{\beta}{2}\right)^{2} \frac{3 ! N}{\left(\frac{\beta N}{2}-1\right)\left(\frac{\beta N}{2}-2\right)(N+1)(N+2)}
$$




\begin{tabular}{|c|c|c|c|c|c|c|c|c|c|c|c|}
\hline & \multicolumn{3}{|c|}{$\beta=1$} & \multicolumn{4}{|c|}{$\beta=2$} & \multicolumn{4}{|c|}{$\beta=4$} \\
\hline$N$ & 2 & 3 & 4 & 2 & 3 & 4 & 5 & 2 & 3 & 4 & 5 \\
\hline$\left\langle q_{1}^{2} q_{2}\right\rangle$ & $\overline{-}$ & - & $\frac{4 \cdot 6 \cdot 1 !}{6 !}$ & $\frac{2 \cdot 2 \cdot 0 !}{4 !}$ & $\frac{2 \cdot 3 \cdot 1 !}{5 !}$ & $\frac{2 \cdot 4 \cdot 2 !}{6 !}$ & $\frac{2 \cdot 5 \cdot 3 !}{7 !}$ & $\begin{array}{l}\frac{4 \cdot 2 \cdot 1 !}{3 \cdot 4 !} \\
\end{array}$ & $\frac{4 \cdot 3 \cdot 2 !}{5 \cdot 5 !}$ & $\frac{4 \cdot 4 \cdot 3 !}{7 \cdot 6 !}$ & $\frac{4 \cdot 5 \cdot 4 !}{9 \cdot 7 !}$ \\
\hline$\left\langle q_{1}^{3} q_{2}\right\rangle$ & - & - & - & & $\frac{2 \cdot 31 \cdot 0 !}{6 !}$ & $\frac{2 \cdot 53 \cdot 1 !}{7 !}$ & $\frac{2 \cdot 81 \cdot 2 !}{8 !}$ & $\frac{4 \cdot 2 \cdot 7 \cdot 0 !}{3 \cdot 5 !}$ & $\frac{4 \cdot 3 \cdot 10 \cdot 1 !}{5 \cdot 6 !}$ & $\frac{4 \cdot 4 \cdot 13 \cdot 2 !}{7 \cdot 7 !}$ & $\frac{4 \cdot 5 \cdot 16 \cdot 3 !}{9 \cdot 8 !}$ \\
\hline$\left\langle q_{1}^{3} q_{2}^{2}\right\rangle$ & - & - & - & - & $\frac{6 \cdot 3 \cdot 7 \cdot 0 !}{7 !}$ & $\frac{6 \cdot 4 \cdot 9 \cdot 1 !}{8 !}$ & $\frac{6 \cdot 5 \cdot 11 \cdot 2 !}{9 !}$ & $\frac{6 \cdot 4 \cdot 4 \cdot 0 !}{3 \cdot 6 !}$ & $\frac{6 \cdot 4 \cdot 9 \cdot 1 !}{5 \cdot 7 !}$ & $\frac{6 \cdot 4 \cdot 16 \cdot 2 !}{7 \cdot 8 !}$ & $\frac{6 \cdot 4 \cdot 25 \cdot 3 !}{9 \cdot 9 !}$ \\
\hline$\left\langle q_{1}^{2} q_{2} q_{3}\right\rangle$ & - & $\frac{2 \cdot 0 !}{5 !}$ & $\frac{2 \cdot 12 \cdot 1 !}{7 !}$ & - & $\frac{2 \cdot 3 \cdot 1 !}{6 !}$ & $\frac{2 \cdot 4 \cdot 2 !}{7 !}$ & $\frac{2 \cdot 5 \cdot 3 !}{8 !}$ & - & $\frac{4 \cdot 3 \cdot 2 !}{5 \cdot 6 !}$ & $\frac{4 \cdot 4 \cdot 3 !}{7 \cdot 7 !}$ & $\frac{4 \cdot 5 \cdot 4 !}{9 \cdot 8 !}$ \\
\hline$\left\langle q_{1}^{2} q_{2}^{2} q_{3}\right\rangle$ & - & - & $\frac{2 \cdot 36 \cdot 1 !}{8 !}$ & - & $\frac{2 \cdot 7 \cdot 1 !}{7 !}$ & $\frac{2 \cdot 9 \cdot 2 !}{8 !}$ & $\frac{2 \cdot 11 \cdot 3 !}{9 !}$ & - & $\frac{8 \cdot 3 \cdot 2 !}{5 \cdot 7 !}$ & $\frac{8 \cdot 4 \cdot 3 !}{7 \cdot 8 !}$ & $\frac{8 \cdot 5 \cdot 4 !}{9 \cdot 9 !}$ \\
\hline
\end{tabular}

TABLE I: Summary of results for other moments for $\beta=1,2,4$, some of which were obtained numerically.

which agrees with those of Ref. 44 for $\beta=2$ in the semiclassical limit, and of Ref. 45 for any $\beta$ and $N$. Both results in Eq. (88) were reported in Refs. 24, 28 for $\beta=1$ and 2. Another case that can be easily obtained from Eqs. (82) and (84), which is of particular interest, is

$$
\left\langle q_{1} q_{2}\right\rangle^{(\beta)}=\frac{1}{N(N+1)}
$$

which was reported in Ref. 29 for $\beta=2$.

Since the Wigner time delay is $\tau_{W}=\tau_{H} \sum_{i}^{N} q_{i} / N$, its mean and variance can be calculated from Eqs. (88) and (90) for any $\beta$ and $N$; the results are

$$
\tau_{W}=\frac{\tau_{H}}{N}, \quad \frac{\left\langle\tau_{W}^{2}\right\rangle-\left\langle\tau_{W}\right\rangle^{2}}{\left\langle\tau_{W}\right\rangle^{2}}=\frac{2}{\left(\frac{\beta N}{2}-1\right)(N+1)},
$$

which for $\beta=2$ reduces to those of Refs. 36,37 .

From Eqs. (57), (62), (63), the second equation in (71), and Eq. (777), is feasible to find that

$$
K_{N}^{(\beta)}(2,2,0, \ldots, 0)=\frac{2 N(2 N+1)}{(N+2)(N+3)} .
$$

Although we do not have explicit results for $\beta=1$, we have verified this expression numerically. This result allows to obtain

$$
\left\langle q_{1}^{2} q_{2}^{2}\right\rangle^{(\beta)}=\left(\frac{\beta}{2}\right)^{4} \frac{\left(\frac{\beta N}{2}-2\right) !}{\left(\frac{\beta N}{2}\right) !} \frac{\left[\frac{\beta(N+1)}{2}-2\right] !}{\left[\frac{\beta(N+1)}{2}\right] !} K_{N}^{(\beta)}(2,2,0, \ldots, 0) .
$$

In Table \ we summarize some moments expressed in Eqs. (58), (65), (66), (80), and others that were obtained numerically. They can easily be generalized to

$$
\left\langle q_{1}^{2} q_{2}\right\rangle^{(\beta)}=\left(\frac{\beta}{2}\right)^{3} \frac{\left(\frac{\beta N}{2}-2\right) !}{\left(\frac{\beta N}{2}\right) !} \frac{\left[\frac{\beta(N+1)}{2}-1\right] !}{\left[\frac{\beta(N+1)}{2}\right] !} K_{N}^{(\beta)}(2,1,0,0, \ldots, 0),
$$

with

$$
\begin{gathered}
K_{N}^{(\beta)}(2,1,0,0, \ldots, 0)=\frac{2 N}{N+2} \\
\left\langle q_{1}^{3} q_{2}\right\rangle^{(\beta)}=\left(\frac{\beta}{2}\right)^{4} \frac{\left(\frac{\beta N}{2}-3\right) !}{\left(\frac{\beta N}{2}\right) !} \frac{\left[\frac{\beta(N+1)}{2}-1\right] !}{\left[\frac{\beta(N+1)}{2}\right] !} K_{N}^{(\beta)}(3,1,0,0, \ldots, 0),
\end{gathered}
$$

where

$$
K_{N}^{(\beta)}(3,1,0,0, \ldots, 0)=\frac{2\left[3 N^{2}+N+(2-\beta / 2)\right]}{(N+3)(N+2)}
$$




$$
\left\langle q_{1}^{3} q_{2}^{2}\right\rangle^{(\beta)}=\left(\frac{\beta}{2}\right)^{5} \frac{\left(\frac{\beta N}{2}-3\right) !}{\left(\frac{\beta N}{2}\right) !} \frac{\left[\frac{\beta(N+1)}{2}-2\right] !}{\left[\frac{\beta(N+1)}{2}\right] !} K_{N}^{(\beta)}(3,2,0,0, \ldots, 0)
$$

with

$$
\begin{gathered}
K_{N}^{(\beta)}(3,2,0,0, \ldots, 0)=\frac{6 N^{2}(2 N+1)}{(N+4)(N+3)(N+2)} \\
\left\langle q_{1}^{2} q_{2} q_{3}\right\rangle^{(\beta)}=\left(\frac{\beta}{2}\right)^{4} \frac{\left(\frac{\beta N}{2}-2\right) !}{\left(\frac{\beta N}{2}\right) !} \frac{\left[\frac{\beta(N+1)}{2}-1\right] !}{\left[\frac{\beta(N+1)}{2}\right] !} \frac{\left[\frac{\beta(N+2)}{2}-1\right] !}{\left[\frac{\beta(N+2)}{2}\right] !} K_{N}^{(\beta)}(2,1,1,0, \ldots, 0),
\end{gathered}
$$

where

$$
K_{N}^{(\beta)}(2,1,1,0, \ldots, 0)=\frac{2 N}{N+3}
$$

and

$$
\left\langle q_{1}^{2} q_{2}^{2} q_{3}\right\rangle^{(\beta)}=\left(\frac{\beta}{2}\right)^{5} \frac{\left(\frac{\beta N}{2}-2\right) !}{\left(\frac{\beta N}{2}\right) !} \frac{\left[\frac{\beta(N+1)}{2}-2\right] !}{\left[\frac{\beta(N+1)}{2}\right] !} \frac{\left[\frac{\beta(N+2)}{2}-1\right] !}{\left[\frac{\beta(N+2)}{2}\right] !} K_{N}^{(\beta)}(2,2,1,0, \ldots, 0)
$$

with

$$
K_{N}^{(\beta)}(2,2,1,0, \ldots, 0)=\frac{2 N(2 N+1)}{(N+4)(N+3)} .
$$

The results of Eqs. (93), (96) and (100) were reported in Ref. 29 for $\beta=2$.

\section{CONCLUSIONS}

We have calculated generalized joint moments of proper delay times for an arbitrary number of channels $N$ and any symmetry $\beta=1,2$ and 4 , which are needed to quantify transport properties, or their fluctuations, through ballistic open systems. This was done by reducing the calculation of the negative moments of the generalized Laguerre distribution to simpler formulas, which are easier to manage analytically. As an important result we show that the $k$ th moment of a proper delay time differs from that of the partial delay time, where the difference comes from the level repulsion of the proper delay times. From our results, also, we were able to generalize the distribution of the partial times to any symmetry. Our general expressions reproduce the existing results for particular cases and those obtained for individual proper and partial delay times. Also, we obtained the mean and variance of the Wigner time delay for arbitraries $N$ and $\beta$, which reproduces the known results for $\beta=2$. Although we regarded perfect coupling of the system to the open channels, we hope that our results encourage further calculations to include an imperfect coupling, as was done for partial delay times.

\section{Acknowledgments}

M. Martínez-Mares and J. C. García are grateful with the Sistema Nacional de Investigadores, Mexico. M. Martínez-

Mares is also grateful with M. A. Torres-Segura for her encouragement. A. M. Martínez-Argüello thanks CONACyT, Mexico for financial support.

\section{Appendix A: Properties associated to $F_{\theta_{n}}$}

There are some relations between the Gamma, Poisson and Negative Binomial probability distributions that are summarized in the following propositions: 
Proposition 1. For $b, b_{1}, b_{2}>0, k, m, n \in \mathbb{Z}_{+}$, and $x \geq 0$,

$$
\begin{aligned}
& F_{n, b}(x)=\int_{0}^{x} f_{n, b}(t) \mathrm{d} t=\sum_{k=n}^{\infty} P_{\text {Poisson }}(b x, k), \\
& \int_{0}^{x} f_{m, b_{2}}(t) P_{\text {Poisson }}\left(b_{1} t, k\right) \mathrm{d} t=N B_{m, p}(k) F_{m+k, b_{1}+b_{2}}(x),
\end{aligned}
$$

where $p=b_{2} /\left(b_{1}+b_{2}\right)$ and $P_{\text {Poisson }}(\lambda, k)$ is the Poisson distribution with parameter $\lambda$ given by

$$
P_{\text {Poisson }}(\lambda, k)=\frac{\lambda^{k}}{k !} \mathrm{e}^{-\lambda}, \quad \text { with } \quad k=0,1, \ldots,
$$

and $N B_{m, p}(k)$ is the Negative Binomial distribution $\frac{52}{2}$ with parameters $m \in \mathbb{N}$ and $p \in[0,1]$ :

$$
N B_{m, p}(k)=\left(\begin{array}{c}
m-1+k \\
m-1
\end{array}\right)(1-p)^{k} p^{m}, \quad \text { with } \quad k=0,1, \ldots
$$

Proof. The proof of (A1) is based on the following identity, which is proved by integrating by parts and induction on $n$ (see Chap. 4, exercise 26, p. 200 of Ref. 51),

$$
\frac{1}{n !} \int_{x}^{\infty} t^{n} \mathrm{e}^{-t} \mathrm{~d} t=\sum_{k=0}^{n} \mathrm{e}^{-x} \frac{x^{k}}{k !}
$$

Therefore, with the change of variables $u=b t$ we have that

$$
F_{n, b}(x)=1-\frac{1}{(n-1) !} \int_{b x}^{\infty} u^{n-1} \mathrm{e}^{-u} \mathrm{~d} u=\sum_{k=n}^{\infty} \frac{(b x)^{k}}{k !} \mathrm{e}^{-b x}=\sum_{k=n}^{\infty} P_{\text {Poisson }}(b x, k) .
$$

To prove (A2), let $p=b_{2} /\left(b_{1}+b_{2}\right)$. Then,

$$
\int_{0}^{x} f_{m, b_{2}}(t) P_{\text {Poisson }}\left(b_{1} t, k\right) \mathrm{d} t=\frac{b_{2}^{m} b_{1}^{k}}{(m-1) ! k !} \int_{0}^{x} t^{m+k-1} \mathrm{e}^{-\left(b_{1}+b_{2}\right) t} \mathrm{~d} t=N B_{m, p}(k) F_{m+k, b_{1}+b_{2}}(x) .
$$

Let

$$
F_{\theta_{n}}^{(n)}(x):=\int_{\Delta_{n}(x)} \prod_{i=1}^{n} f_{a_{i}, b_{i}}\left(t_{i}\right) \mathrm{d} t_{i},
$$

where $\Delta_{n}(x)=\left\{\left(t_{1}, \ldots, t_{n}\right): 0 \leq t_{1} \leq t_{2} \leq \cdots \leq t_{n} \leq x\right\}$. By the Cavallieri Principle, the functions $F_{\theta_{n}}^{(n)}(x)$ satisfy the recurrence relation

$$
F_{\theta_{n}}^{(n)}(y)=\int_{0}^{y} f_{a_{n}, b_{n}}(x) F_{\theta_{n-1}}^{(n-1)}(x) \mathrm{d} x .
$$

Also, for this function we have the following properties:

Proposition 2. If $n \geq 2$, all the $a_{i} \in \mathbb{N}, g_{k}:=\sum_{i=1}^{k} b_{i}$, and $p_{k}:=b_{k} / g_{k}$, then

$$
\begin{aligned}
F_{\theta_{1}}^{(1)}(y) & =\sum_{k=a_{1}}^{\infty} P_{\text {Poisson }}\left(b_{1} y, k\right), \quad F_{\theta_{1}}=1, \\
F_{\theta_{n}}^{(n)}(x) & =\sum_{\mathbf{A}^{(n-1)}} \prod_{j=2}^{n} N B_{a_{j}, p_{j}}\left(L_{j-1}\right) F_{a_{n}+L_{n-1}, g_{n}}(x), \\
F_{\theta_{n}} & =\sum_{\mathbf{A}^{(n-1)}} \prod_{j=2}^{n} N B_{a_{j}, p_{j}}\left(L_{j-1}\right)
\end{aligned}
$$

where $L:=\sum_{i=1}^{n} \ell_{i}$, for $\ell_{i} \in \mathbb{N}, \sum_{\mathbf{A}^{(n-1)}}$ means summation over all $\ell_{i} \in \mathbf{A}^{(n-1)}$, with

$$
\mathbf{A}^{(n)}:=\left\{\ell_{1}, \ldots, \ell_{n}: \ell_{i} \geq a_{i}, \ell_{i} \in \mathbb{N}, i=1, \ldots, n\right\} .
$$


Proof. The first formula in (A10), is exactly (A1) of Proposition 1. The second is clear since $F_{\theta_{1}}^{(1)}$ is a probability distribution function. To prove (A11), we proceed by induction on $n$. For $n=2$, (A11) is a direct consequence of Proposition 1, since $a_{1} \in \mathbb{N}$, and the Monotone Convergence Theorem to interchange the series and the integral. As inductive hypothesis, let us assume that (A11) holds for $n-1$, with some $n \geq 3$, and try to prove it for $n$. With this hypothesis we write Eq. (A9) as

$$
\begin{aligned}
F_{\theta_{n}}^{(n)}(y) & =\sum_{\mathbf{A}^{(n-2)}} \prod_{j=2}^{n-1} N B_{a_{j}, p_{j}}\left(L_{j-1}\right) \int_{0}^{y} f_{a_{n}, b_{n}}(x) F_{a_{n-1}+L_{n-2}, g_{n-1}}(x) \mathrm{d} x \\
& =\sum_{\mathbf{A}^{(n-2)}} \prod_{j=2}^{n-1} N B_{a_{j}, p_{j}}\left(L_{j-1}\right) \sum_{k=a_{n-1}+L_{n-2}}^{\infty} N B_{a_{n}, p_{n}}(k) F_{a_{n}+k, g_{n}}(y),
\end{aligned}
$$

where at the last equality we first used (A1) and then (A2) with $m=a_{n}, k=L_{n-2}, p=p_{n}$, taking into account that $p_{n}=b_{n} /\left(b_{n}+g_{n-1}\right)$. If we make the change of variables $\ell_{n-1}=k-L_{n-2}$ we write the last equation as

$$
\begin{aligned}
F_{\theta_{n}}^{(n)}(y) & =\sum_{\mathbf{A}^{(n-2)}} \prod_{j=2}^{n-1} N B_{a_{j}, p_{j}}\left(L_{j-1}\right) \sum_{\ell_{n-1}=a_{n-1}}^{\infty} N B_{a_{n}, p_{n}}\left(\ell_{n-1}+L_{n-2}\right) F_{a_{n}+\ell_{n-1}+L_{n-2}, g_{n}}(y) \\
& =\sum_{\mathbf{A}^{(n-1)}} \prod_{j=2}^{n} N B_{j, p_{j}}\left(L_{j-1}\right) F_{a_{n}+L_{n-1}, g_{n}}(y) .
\end{aligned}
$$

At the last step we used that the sets $\mathbf{A}^{(n)}$ are cartesian products [see [A13)] in fact,

$$
\mathbf{A}^{(n-1)}=\mathbf{A}^{(n-2)} \times\left\{a_{n-1}, 1+a_{n-1}, 2+a_{n-1}, \ldots\right\}
$$

This finishes the proof of A11.

The proof of (A12) is based on the Monotone Convergence Theorem. Indeed,

$$
F_{\theta_{n}}=\lim _{x \rightarrow \infty} F_{\theta_{n}}^{(n)}(x)=\sum_{\left.\mathbf{A}^{(n-1)}\right)} \prod_{j=2}^{n} N B_{a_{j}, p_{j}}\left(L_{j-1}\right) \lim _{x \rightarrow \infty} F_{a_{n}+L_{n-1}, g_{n}}(x)=\sum_{\mathbf{A}^{(n-1)}} \prod_{j=2}^{n} N B_{a_{j}, p_{j}}\left(L_{j-1}\right)
$$

Remark 3. It is not necessary, for Proposition 0 to hold, that the $b_{i}$ 's to belong to $\mathbb{N}$, just that they are positive.

${ }^{1}$ P. W. Brouwer, K. M. Frahm, and C. W. J. Beenakker, Phys. Rev. Lett. 78, 4737 (1997).

${ }^{2}$ P. W. Brouwer, K. M. Frahm, and C. W. J. Beenakker, Waves Random Media 9, 91 (1999).

3 F. T. Smith, Phys. Rev. 118, 349 (1960).

4 E. P. Wigner, Phys. Rev. 98145 (1955).

${ }^{5}$ P. A. Mello and N. Kumar, Quantum Transport in Mesoscopic Systems: Complexity and Statistical Fluctuations (Oxford University Press, New York, 2005).

6 J. Feist, O. Zatsarinny, S. Nagele, R. Pazourek, J. Burgdörfer, X. Guan, K. Bartschat, and B. I. Schneider, Phys. Rev. A 89, 033417 (2014).

7 I. A. Ivanov and A. S. Kheifets, Phys. Rev. A 89, 043405 (2014).

8 P. C. Deshmukh, A. Mandal, S. Saha, A. S. Kheifets, V. K. Dolmatov, and S. T. Manson, Phys. Rev. A 69, 053424 (2014).

9 A. Chacon, M. Lein, and C. Ruiz, Phys. Rev. A 89, 053427 (2014).

10 M. Schultze, M. Fie $\beta$, N. Karpowicz, J. Gagnon, M. Korbman, M. Hofstetter, S. Neppl, A. L. Cavalieri, Y. Komninos, Th. Mercouris, C. A. Nicolaides, R. Pazourek, S. Nagele, J. Feist, J. Burgdörfer, A. M. Azzeer, R. Ernstorfer, R. Kienberger, U. Kleineberg, E. Goulielmakis, F. Krausz, and V. S. Yakovlev, Science 328, 1658 (2010).

11 P. A. Mello in Mesoscopic Quantum Physics, edited by E. Akkermans, G. Montambaux, J.-L. Pichard, and J. Zinn-Justin (Elsevier, Amsterdam, 1995).

12 C. W. J. Beenakker, Rev. Mod. Phys. 69, 731 (1997).

13 Y. Alhassid, Rev. Mod. Phys. 72, 895 (2000).

14 Y. V. Fyodorov, D. V. Savin, and H.-J. Sommers, J. Phys. A: Math. Gen 38, 10731 (2005).

15 B. D. Simons and B. L. Altshuler, Phys. Rev. B 48, 5422 (1993). 
16 Y. V. Fyodorov, Phys. Rev. Lett. 73, 2688 (1994).

17 N. Taniguchi, A. Hashimoto, B. D. Simons, and B. L. Altshuler, Europhys. Lett. 27, 335 (1994).

18 Y. V. Fyodorov and A. D. Mirlin, Phys. Rev. B 51, 13403 (1995).

19 C. M. Marcus, A. J. Rimberg, R. M. Westervelt, P. F. Hopkins, and A. C. Gossard, Phys. Rev. Lett. 69, 506 (1992).

${ }^{20}$ M. W. Keller, A. Mittal, J. W. Sleight, R. G. Wheeler, D. E. Prober, R. N. Sacks, H. Shtrikmann, Phys. Rev. B 53, R1693 (1996).

21 I. H. Chan, R. M. Clarke, C. M. Marcus, K. Campman, A. C. Gossard, Phys. Rev. Lett. 74, 3876 (1995).

22 A. G. Huibers, S. R. Patel, C. M. Marcus, P. W. Brouwer, C. I. Duruöz, and Jr J. S. Harris, Phys. Rev. Lett 81, 1917 (1998).

${ }^{23}$ P. W. Brouwer, S. A. van Langen, K. M. Frahm, M. Büttiker, and C. W. J. Beenakker, Phys. Rev. Lett. 79, 913 (1997).

24 M. Martínez-Mares and E. Castaño, Phys. Rev. E 71, 036201 (2005).

25 M. Switkes, C. M. Marcus, K. Campman and A. C. Gossard, Science 283, 1905 (1999).

26 P. W. Brouwer, Phys. Rev. B 58, R10135 (1998).

27 H. Schanze, H.-J. Stöckmann, M. Martínez-Mares, and C. H. Lewenkopf, Phys. Rev. E 71, 016223 (2005).

28 M. Martínez-Mares, Phys. Rev. E. 72, 036202 (2005).

29 M. Martínez-Mares, C. H. Lewenkopf, and E. R. Mucciolo, Phys. Rev. B 69, 085301 (2004).

30 V. A. Gopar, P. A. Mello, and M. Büttiker, Phys. Rev. Lett. 77, 3005 (1996).

31 Y. V. Fyodorov, D. V. Savin, and H.-J. Sommers, Phys. Rev. E. 55, R4857 (1997).

32 A. Ossipov, T. Kottos, and T. Geisel, Europhys. Lett. 62, 719 (2003).

33 A. Ossipov and Y. V. Fyodorov, Phys. Rev. B 71, 125133 (2005).

34 T. Kottos and M. Weiss, Phys. Rev. Lett. 89, 056401 (2002).

35 J. A. Méndez-Bermúdez and T. Kottos, Phys. Rev. E 72, 064108 (2005).

${ }^{36}$ Y. V. Fyodorov and H.-J. Sommers, Phys. Rev. Lett. 76, 4709 (1996).

37 Y. V. Fyodorov and H.-J. Sommers, J. Math. Phys. 38, 1918 (1997).

38 P. Šeba, K. Życzkowski, and J. Zakrzewski, Phys. Rev. E 54, 2438 (1996).

39 C. Texier and A. Comtet, Phys. Rev. Lett. 82, 4220 (1999).

40 A. Ossipov, T. Kottos, and T. Geisel, Phys. Rev. B 61, 11411 (2000).

41 D. V. Savin, Y. V. Fyodorov, and H.-J. Sommers, Phys. Rev. E 63, 035202(R), (2001).

42 H.-J. Sommers, D. V. Savin, and V. V. Sokolov, Phys. Rev. Lett. 87, 094101 (2001).

43 M. Marciani, P. W. Brouwer, and C. W. J. Beenakker, Phys. Rev B 90, 045403 (2014).

44 G. Berkolaiko and J. Kuipers, J. Phys. A: Math. Theor. 43, 035101 (2010).

45 F. Mezzadri and N. J. Simm, J. Math. Phys. 52, 103511 (2011).

${ }^{46}$ F. Mezzadri and N. J. Simm, J. Math. Phys. 53, 053504 (2012).

47 F. J. Dyson, J. Math. Phys. (N.Y.) 3, 140 (1962).

${ }^{48}$ I. S. Gradshteyn and I. M. Ryzhik, Table of Integrals, Seriesm and Products (Academic Press, Inc., Orlando, 1980$)$, p. 1111.

49 G. Birkhoff and S. Mac Lane, A survey of Modern Algebra (AKP Classics, U.S.A, 1998).

50 I. N. Herstein, Topics in Algebra (John Wiley \& Sons, 2nd Edition, New York, 1975).

51 S. Ross, A First Course in Probability (Pearson Prentice Hall, 7th Edition, New Jersey, 2006).

52 Handbook of Mathematical Functions, ed. by M. Abramowitz and I. A. Stegun (Dover Publications, Inc., New York, 1972).

${ }^{53}$ The negative binomial distribution can be used only when $N$ is an even number. 